\title{
Demand for long acting and permanent methods of contraceptives and factors for non-use among married women of Goba Town, Bale Zone, South East Ethiopia
}

Abulie Takele ${ }^{1 *}$, Getu Degu ${ }^{2}$ and Mezgebu Yitayal ${ }^{3}$

\begin{abstract}
Background: Contraceptive use including short acting, long acting and permanent methods positively influence the socio-economic development of a nation by allowing families to space and limit their family size to their economic capacity. Demand for LAPMs of contraception as detrmined by utilization and unmet need for LAPMs of contraception can provide realiable information for providers.

Objective: To determine the utilization of long acting and permanent contraception and its associated factors among married women of Goba town, South East Ethiopia.

Methods: A cross sectional community based study was conducted among 734 systematically selected married women of reproductive age in Goba town in September/ 2009. A structured and pretested, interview questionaire was used to collect data on socio-demographic, behavioral factors and data related to demand for LAPMs of contraception. Data were analyzed using EPI INFO and SPSS version 16.

Result: The demand for Long Acting and Permanent Methods (LAPMs) of contraception was $18.1 \%$. Utilization of LAPMs of contraception in the town was 64 (8.7\%) and the unmet need for LAPMs was 69 (9.4\%). Information on LAPMs in the town was 636 (86.6\%). Media (radio and television) was the major sources of information 641 (87.3\%).

The use of LAPMs was significatly associated with ever use AOR[17.43, 95\% Cl:9.19, 33.03], number of times discussions made on methods AOR[4.6, 95\% Cl: 1.72,12.17] and main decider of using methods AOR[ 2.2, 95\% Cl:1.03, 4.65]. It was not associated with socio-demographic variables.

Conclusion and recommendation: The utilization of LAPMs in the town was less although higher than the Ethiopian demographic and health survey 2005 result. Moreover, there was a considerable unmet need. Increase the method mix of LAPMs by incorporating varaies of implnats in order to increase utilization. Proper counseling of client and partners discussion were some of the recommendation forwarded.
\end{abstract}

Keywords: Long Acting and Permanent Methods, Demand, Unmet need, Utilization, Implants, Contraception

\footnotetext{
* Correspondence: abuletakele@yahoo.com

'College of Medicine \& Health Sciences, Madawalabu University, Robe, Bale, Ethiopia

Full list of author information is available at the end of the article
} 


\section{Background}

The international conference on population and development held in Cairo in 1994, drew the attention of international community and national governments to the need to improve reproductive health services especially in low economic status countries like Ethiopia [1].

Reproductive-age women have varying contraceptive needs. For many women, long-term reversible contraception, defined as Intra Uterine Devices (IUDs) and implants represents an excellent choice [2-4]. Researchers have showen that many women do not use oral contraceptives effectively. One million pregnancies result from the faulty use of oral contraceptives each year; in addition, unintended pregnancies remain common. Clearly, the method of contraception must tailor to meet the needs of such individuals $[3,4]$. Long-acting and Permanent Methods of Contraception (LAPMCs) remain relatively small and sometimes missing component of national Family Planning(FP) programs in Sub Saharan Africa (SSA). These methods can enhance FP programs in meaningful ways if the challenges to their availability, access, and acceptability can be overcome [3].

Family Planning service that provides accurate and complete information about contraceptive methods meets the need of their clients. Still, early marriage and producing too many children, which are close to each other is a common practice of developing countries including Ethiopia. The total fertility rate (TFR) is 4.8 nationally and 5.6 in Oromia region. Long Acting and Permanent Methods of contraception use have lowest rates. It account IUD $0.3 \%$; implant $3.4 \%$ and female sterilization $0.5 \%$. The contraceptive prevalence rate at national level was $28.6 \%$. The unmet need for family planning was $25 \%$ [2]. Hormonal implants are frequently under-utilized FP methods [3,4]. Knowledge of LAPMCs in Oromia region was IUD $12.2 \%$, Implants $20 \%$ and Tubal ligation 17.2\%. Ever use and current use of Long Acting and Permanent Methods (LAPMs) of contraception in oromia region was less than one percent [5].

Determining the utilization and unmet need for LAPMs of contraceptives indicates demand for contraception by indicating potential users of LAPMs of contraceptives. In the study area, separate study was not conducted to determine demand for LAPMs contraceptives. In order to address this problem it is important to study the factors associated and make the services available at the lowest possible level. Moreover, this study focuses on assessment of the demand for LAPMs of contraceptives and factors for nonutilization among married women of reproductive age in Goba town.

\section{Methodology}

\section{Study setting and sample}

The study was conducted in Bale Zone Goba Town located at $450 \mathrm{~km}$ from Addis Ababa in the South East Ethiopia. With a total area of $1619.38 \mathrm{sq} . \mathrm{km}$, population of 32,916 , population density of 20.34 people per sq. $\mathrm{km}$ and household size of 4.2 people per household and 7,837 household in two sub-cites. A cross sectional community based study conducted in Goba town in September 2009 to assess the demand of LAPMs contraception and factors for non-use among married women of Goba town. The source population was all married women found in Goba town aged 15-49 years. The study population was married women aged 15-49 years found in the three randomly selected Kebeles of Goba town.

A sample of 734 married women of reproductive age was calculated using a proportion of $50 \%$, a type 1 error $5 \%$, a power of $80 \%$ and a design effect of two. The town was divided in to six kebele and three of six kebeles were selected by lottery method. Considering at least there is one married woman present per household, and using the number of households as sampling frame. Systematic random sampling method used to take proportional numbers of women from three kebeles. Interview was made by face to face interviewing of married women from the selected kebeles.

\section{Measurements}

A structured and pre- tested interview based questionnaire was used to collect the quantitative data. The questionnaire was comprised of questions for assessing socio-demographic, social, behavioral and service factors were prepared. Some of the questions were taken from EDHS and modified. Trained diploma holders female data collectors collected the data by interviewing wives in the three kebeles. Questionnaire was first prepared in English and translated to Amharic to make it understandable by the study subjects. Data collectors explained the purpose of the study and obtaining verbal consent from the respondent. The data collectors have checked for completeness and consistency before leaving the interviewee.

\section{Data analysis}

Data was analyzed using EPI-INF 6 and exported to SPSS version 16. Recoding and transforming of some data (age of respondent, number of pregnancy, number of children alive, religion, educational status, occupation, monthly income, husband opinion on partners using LAPMs of contraceptives, number of times discussion on LAPMs and main decider on using LAPMs of contraceptives) was done to make them categorical. Descriptive statistics computed to determine the prevalence of LAPMs of contraception 
use. Analysis such as proportion, percentage, frequency distribution, statistical methods logistic regression, odds ratio with 95\% confidence interval, and P-Value used in describing the data. The results were displayed using tables. Association of socio-demographic and other variables were determined using cross tabulating, and odds ratio (OR) with $95 \%$ confidence intervals. Stepwise logistic regression was used to control for confounding.

\section{Ethical clearance}

Letter of ethical clearance was obtained from Gondar University School of Public Health Ethical Research Committee. Formal letter of cooperation was written from Gondar University School of Public Heath to Goba town administrative council. Verbal consent was obtained from the respondents and response of participants was anonymous and confidential.

For uniformity of understanding of the approach of key words is given below

- Demand for LAPMs:- The sum of LAPM being used (Met need) and method that is desired but not used due to any reason (unmet need).

- Long acting contraceptive: - Intra-Uterine Device (IUD) and Norplant.

- Permanent methods of contraceptive- Tubal ligation

- Unmet need for LAPMs of Contraceptive: - A condition of wanting to postpone or avoid pregnancy but not using any of the LAPMs.

- Unmet need for LAPMs of contraceptive during pregnancy:- A phrase to describe a woman who is pregnant at the time of the study but whose pregnancy was not intended.

- Unmet need for limiting:- When women who do not want any more children, but not using any of LAPM or others methods.

- Unmet need for spacing:- When a woman who does not want to have pregnancy soon after delivery or want to space for two years but not using any of LAPM or other methods.

\section{Results}

\section{Socio-demographic characteristics of the respondents}

The response rate for the study was $100 \%$. The mean age of the respondents was $30 \pm 7.1$ S.D and the median age was 29 years. Majority of the respondents 596 (81.2\%) were in the age group 20-39 years. Three hundred forty three $46.7 \%$ of the respondents had high school education, 32 (4.4\%) have informal education and $44(6 \%)$ have no education. The ethnicity of the respondents was Oromo 350 (47.7\%) followed by Amhara 336 (45.8\%). The religion of most respondents was Orthodox 490 (66.8)\%, followed by Muslim161 (21.9\%). The occupation of respondents was
442 (60.2\%) house wife followed by government employee 135 (18.4\%). The monthly income of the majority of the respondents were in the second (30.5\%) was in the middle class (32.0\%). Income level was classified by taking the minimum current Government employee salary as the first class (Table 1). Four hundred ninety nine $(65.7 \%)$ of the respondents have more than five hundred birr per month.

\section{Utilization of LAPMs of contraceptives}

The current utilization rate of LAPMs of contraceptives in the town was $64(8.72 \%)$. Of these 48 (6.5\%) were

\section{Table 1 Socio-demographic characteristics of married} women of Goba town, South East Ethiopia, September/ 2009

\begin{tabular}{lll}
\hline Variable & Number & Percent \\
\hline Age of the women & 32 & \\
\hline $15-19$ & 136 & 4.4 \\
\hline $20-24$ & 210 & 18.5 \\
\hline $25-29$ & 140 & 28.6 \\
\hline $30-34$ & 110 & 19.1 \\
\hline $35-39$ & 66 & 15.0 \\
\hline $40-44$ & 40 & 9.0 \\
\hline $45-49$ & & 5.4 \\
\hline
\end{tabular}

Educational status

\begin{tabular}{lll}
\hline Illiterate & 44 & 6.0 \\
\hline Able to read /write & 32 & 4.4 \\
\hline Elementary & 148 & 20.2 \\
\hline High school & 343 & 46.7 \\
\hline 12 and above & 167 & 22.7 \\
\hline
\end{tabular}

Ethnicity

\begin{tabular}{lll}
\hline Oromo & 350 & 47.7 \\
\hline Amhara & 336 & 45.8 \\
\hline Gurage & 29 & 4.0 \\
\hline Tigre & 13 & 1.8 \\
\hline Others $^{*}$ & 6 & 0.8
\end{tabular}

\section{Religion}

\begin{tabular}{lll}
\hline Muslim & 161 & 21.9 \\
\hline Orthodox & 490 & 66.8 \\
\hline Protestant & 74 & 10.1 \\
\hline Catholic & 6 & 0.8 \\
\hline Others** & 3 & 0.4
\end{tabular}

\section{Occupation}

\begin{tabular}{lll}
\hline Government Employee & 135 & 18.4 \\
\hline House wife & 442 & 60.2 \\
\hline Merchant & 104 & 14.2 \\
\hline Daily laborer & 14 & 1.9 \\
\hline Student & 36 & 4.9 \\
\hline Others*** & 3 & 0.4
\end{tabular}

${ }^{*}=$ Sidama, kembata, somali ${ }^{* *}=$ Kalhiwot, Missionary ${ }^{* *}=$ Tella sailors, self employee002Ccpe. 
using Norplant, 11 (1.5\%) IUD and 5 (0.7\%) TL. One hundred thirty six (18.5\%) of the respondents had ever used LAPMs. The methods ever used were 94 (12.8\%) Norplant, 37 (5.0\%) IUD and 5 (0.7\%) TL. Two hundred nineteen $29.8 \%$ were pregnant and 195 (26.2\%) had five or more pregnancies.

The utilization of LAPMs of contraceptives varies with change in the age group of respondents. The highest frequency of use was observed in the age group 25-29 years of age. However, there was a decrease in use of LAPMs as age of the women increase from 30-49 years.

Two hundred thirty nine $32.6 \%$ had no child and 155 (21.1\%) had five and more than five children alive. Among $64(8.72 \%)$ of current LAPMs users thirty-five $54.7 \%$ of users were using LAPMs of contraceptives for delaying pregnancy while twenty-six $40.6 \%$ of users were using LAPMs they did not want any more baby (Table 2).

\section{Demand and unmet need for LAPMs}

The total demand for LAPMs of contraceptives in the town was found to be $18.12 \%$. The unmet need for LAPMs of contraceptives was sixty-nine 9.4\% (3.78\% for spacing and $5.59 \%$ for limiting). Four $0.55 \%$ respondents who were pregnant at the time of the study had past unmet need as they have unintended current pregnancy of

Table 2 Percent distribution of current use, unmet need and demand for LAPMs by age, educational status and religion of married women of Goba town, South East Ethiopia, September/ 2009

\begin{tabular}{|c|c|c|c|}
\hline Variables & Using LAPMs \% & Unmet need \% & Demand \% \\
\hline \multicolumn{4}{|l|}{ Age } \\
\hline $15-19$ & 0.0 & 0.3 & 0.3 \\
\hline $20-24$ & 0.5 & 1.1 & 1.6 \\
\hline $25-29$ & 3.0 & 2.6 & 5.6 \\
\hline $30-34$ & 1.6 & 1.4 & 3.0 \\
\hline $35-39$ & 1.8 & 2.0 & 3.8 \\
\hline $40-44$ & 1.2 & 1.4 & 2.6 \\
\hline $45-49$ & 0.5 & 0.7 & 1.2 \\
\hline \multicolumn{4}{|l|}{ Educational status } \\
\hline Illiterate & 0.1 & 0.4 & 0.5 \\
\hline Able to read /write & 0.4 & 0.5 & 0.9 \\
\hline Elementary & 1.1 & 1.6 & 2.7 \\
\hline High school & 4.5 & 3.8 & 8.3 \\
\hline 12 and above & 2.6 & 3.0 & 5.6 \\
\hline \multicolumn{4}{|l|}{ Religion } \\
\hline Muslim & 1.6 & 1.6 & 3.3 \\
\hline Orthodox & 5.3 & 6.9 & 10.9 \\
\hline Protestant & 1.5 & 0.8 & 2.3 \\
\hline Catholic & 0.3 & 0.0 & 0.3 \\
\hline Others & 0.0 & 0.0 & 0.0 \\
\hline
\end{tabular}

which two $(0.27 \%)$ want spacing and two $(0.27 \%)$ want limiting. Eighty-seven (11.8\%) of the respondents want to limit their number of children. Of these 20 (2.72\%) of them prefer to use LAPMs of contraception and were having unmet need for limiting. Four hundred ten $55.8 \%$ of the respondents were using short acting contraceptives including natural methods. Nevertheless, 33 (4.5\%) of them did not want to use the method that they were using.

The total unmet need was higher in the age group 2529 Years and it decline from age of 35 to 49 years. In addition, it was observed that there was declining of unmet need between ages 30-34 years. The unmet need for limiting increases with age, as age increase from 15 to 29 years. There was a decrease in pattern of unmet need for limiting and spacing as age increase from 3049 years of age (Table 2).

\section{Associated factors utilization and unmet need for LAPMs}

Two third (66.9\%) of the respondents ever heard at least one LAPMs of contraceptives. The most ever heard LAPMs of contraceptive was Norplant 641 (87.3\%). Four hundred forty five $(60.8 \%)$ of the respondents have discussed with health professional about LAPMs. Six hundred thirty eight $86.9 \%$ reported, they knew where LAPMs of contraceptives were found in the town. Six hundred thirty six (86.6\%) of the respondents have information about LAPMs from different sources. The major source of information was media 641 (87.3\%) (Radio and Television) (Table 3 ). In the past few months, only two hundred fifty one $34.2 \%$ of the respondents discussed about LAPMs. Moreover, 179 (71.3\%) have discussed with their husband and 90 (35.9\%) discussed with their friends/neighbor. Three hundred fifteen (42.9\%) of the respondents had never discussed about LAPMs of contraceptives with their husband /partner. Four hundred ninthly six $(67.6 \%)$ of the respondents responded that their husband/partner approves partners using LAPMs. The decision of using LAPMs of contraceptive was mainly made by both partners together for 474 (64.6\%) of the respondents (Table 3).

Among 677 (92.2\%) who were not pregnant and not sure of their pregnancy status in the study period, 351 (51.9\%) wanted to become pregnant latter and 253 (37.4\%) did not want any more. Of these seventy-five (21.4\%) want to delay their next baby prefer to use LAPMs. The rest ninety (35.6\%) want to use LAPMs to limit number of children. For majority of the respondents 532 (79.9\%) their last pregnancy was intentional. Among current short acting contraceptive users 24 (68.6\%) prefer to use Norplant. For half $12(50.0 \%)$ of the non-users of Norplant the reason for not using Norplant was fear of complications. For those who want to use LAPMs, but are not using the methods they prefer the reasons for non-use are previous 
Table 3 Percentage of ever heard, intention to use, Knowledge of source, discussion with husband and source of information of LAPMs of married women of Goba town, South East Ethiopia September/ 2009

\begin{tabular}{|c|c|c|c|}
\hline Variables & Response & Frequency & Percent \\
\hline \multirow[t]{3}{*}{ Ever heard at least LAPMs } & IUD & 491 & 66.9 \\
\hline & Norplant & 641 & 87.3 \\
\hline & Tubal ligation & 323 & 44.0 \\
\hline \multirow[t]{2}{*}{ Discussed with $\mathrm{h} /$ professional LAPMs } & Yes & 289 & 39.4 \\
\hline & No & 445 & 60.8 \\
\hline \multirow[t]{2}{*}{ Knows source of LAPMs } & Yes & 638 & 86.9 \\
\hline & No & 96 & 13.1 \\
\hline \multirow[t]{4}{*}{ Mentioned sources LAPMs } & Hospital & 636 & 99.7 \\
\hline & Health center & 17 & 2.6 \\
\hline & Clinic & 12 & 1.9 \\
\hline & Private health facility & 10 & 1.6 \\
\hline \multirow[t]{2}{*}{ Heard information of LAPMs } & Yes & 636 & 86.6 \\
\hline & No & 98 & 13.4 \\
\hline \multirow[t]{6}{*}{ Source of information of LAPMs } & Radio & 389 & 53.0 \\
\hline & Television & 252 & 34.3 \\
\hline & News paper & 60 & 8.2 \\
\hline & Pamphlet & 66 & 9.0 \\
\hline & Community events & 488 & 66.5 \\
\hline & Health personnel & 24 & 32.7 \\
\hline \multirow[t]{3}{*}{ Discussed with husband on LAPMs } & Not at all & 315 & 42.9 \\
\hline & Once/twice & 222 & 30.2 \\
\hline & More often & 197 & 26.8 \\
\hline \multirow[t]{3}{*}{ Husband attitude to LAPMs } & Approves & 496 & 67.6 \\
\hline & Opposes & 38 & 5.2 \\
\hline & Don't know & 200 & 27.2 \\
\hline \multirow[t]{3}{*}{ Main decider on using LAPMs } & self & 218 & 29.7 \\
\hline & Husband & 42 & 5.7 \\
\hline & Both decide together & 474 & 64.6 \\
\hline
\end{tabular}

method inconvenient, health problem, husband/partner opposes and fear of complications (Table 4).

In the last few months only two hundred fifty one $34.2 \%$ of the respondents discussed about LAPMs. Discussion was made with husband/partner 179 (71.3\%), father/ mother $4(1.6 \%)$, daughter $7(2.8 \%)$, health professionals 48 (19.1\%) and friend/neighbor 90 (35.9\%). Three hundred fifteen (42.9\%) of the respondents had never discussed about LAPMs of contraceptives with their husband /partner. Four hundred ninety six (67.6\%) of the respondents responded that their husband/partner approves partners using LAPMs. Thirty-eight 5.2\% the decision of using LAPM of contraceptive was mainly made by both partners together for 474 (64.6\%) of the respondents (Table 4).

Among those who were not pregnant and not sure 677 (92.2\%) in the study period 351(51.9\%) wanted to become pregnant latter and $253(37.4 \%)$ did not want any more.
Of seventy five (21.4\%) who want to delay their next baby prefer to use LAPMs and ninety (35.6\%) want to use LAPMs to limit number of children (Table 4).

Majority of the respondents $543(80.2 \%)$ their last pregnancy was intentional. Among current short acting contraceptive users 24 (68.6\%) preferred to use Norplant and for half 12 (50\%) of the respondents reason for not using Norplant was fear of complications. IUD was not used in the past because of proven health problem 4 (57\%) (Table 5).

In order to see the association between unmet need for LAPMs and explanatory variables, binary logistic regression carried out to assess the statistical association of the dependent variable and the covariates. Those variable found to be statistically significant within the binary logistic regression model based on the COR and their pvalue ( $p<0.05$ ), were entered to the multivariable logistic regression model so that to come up with final 
Table 4 Percentage of pregnancy status, plan for next baby, and proffered method, reason for none use of methods of married women of Goba town, South East Ethiopia, September 2009

\begin{tabular}{|c|c|c|c|}
\hline Variable & Response & Number & Percen \\
\hline & Yes & 57 & 7.8 \\
\hline \multirow[t]{2}{*}{ Currently pregnant $n=734$} & No & 669 & 91.1 \\
\hline & Not sure & 8 & 1.1 \\
\hline \multirow[t]{2}{*}{ Pregnancy planned $n=57$} & Yes & 50 & 87.7 \\
\hline & No & 7 & 12.3 \\
\hline \multirow[t]{3}{*}{ Next baby is planned $n=677$} & Latter & 351 & 51.9 \\
\hline & Soon & 73 & 10.8 \\
\hline & Not any more & 253 & 37.4 \\
\hline \multirow[t]{2}{*}{ Preferred method to space $n=351$} & Short Acting & 276 & 78.6 \\
\hline & LAPMs & 75 & 21.4 \\
\hline \multirow[t]{3}{*}{ Preferred method limiters $n=253$} & Short Acting & 143 & 56.5 \\
\hline & LAPMs & 90 & 35.6 \\
\hline & Nothing & 20 & 7.9 \\
\hline \multirow[t]{2}{*}{ Want TL for limiters $n=253$} & Yes & 29 & 11.5 \\
\hline & No & 224 & 88.5 \\
\hline \multirow[t]{5}{*}{ Reasons for not having TL $n=224$} & Intercourse related & 46 & 20.6 \\
\hline & Opposition of Tubal ligation & 91 & 40.6 \\
\hline & Method related & 23 & 10.3 \\
\hline & Health concern & 43 & 19.2 \\
\hline & Prefer short acting methods & 36 & 16.1 \\
\hline
\end{tabular}

predictors of utilization and unmet need of LAPMs contraception with their respective adjusted odds ratio. Ever use of LAPMs of contraceptives was found to be a strong predictor of current use of LAPMs, respondents who had ever used LAPMs of contraception were more than seventeen times more likely to use LAPMs than those who did not have ever used LAPMs $[\mathrm{AOR}=17.43$, 95\% CI:9.19, 33.03]. The number of times discussion on LAPMs was made was also found to be predictor of using LAPMs of contraceptives, respondents who discussed more often were more than four times more likely to use LAPMs than those who have discussed once or twice about LAMPs of contraceptives [AOR=4.6, 95\% CI:1.72, 12.17]. Statistically significant association was not seen between age of women, number of children alive, educational status, religion, income of the family and other variables; and utilization of LAPMs of contraceptives (Table 6).

The intention to use LAPMs of contraceptives in the future was found to be a predictor of unmet need for LAPMs, respondents who were intending to use LAPMs were found about four times more likely to have had unmet need for LAPMs of contraceptives than those who did not intend to use [AOR=3.99, 95\% CI:2.27, 7.02]. The other important predictor found was number of pregnancy, respondents who had five or more pregnancies were more than three times more likely to have unmet need than those who had not ever been pregnant $[\mathrm{AOR}=3.67,95 \% \mathrm{CI}: 1.75,7.71]$. The other sociodemographic and behavioral factors were not found to have statistically significant association with unmet need for LAPMs of contraceptives (Table 7).

\section{Discussion}

Information about LAPMs of contraceptives was high $86.6 \%$. In Gondar town information of modern contraception was 74.9\% [6] In EDHS 2011, the information about over all modern contraception was $97.4 \%$ the difference may be due to increased awareness of communities about contraception including LAPMs in the through time [2]. Having information on more than one LAPMs allows clients to choose the preferred method to use. The method mix of LAPMCs is required to increase the use rate of LAPMs [7]. The major source of information, media in this study could create an opportunity to transfer information [8]. The utilization rate of LAPMs of contraception in the town was $64(8.7 \%)$. This was still small proportion of the utilization of overall modern contraception in the town $59.1 \%$. This is in line with the DHS survey result in Kenya [9]. In other sub Saharan African countries the utilization rate of LAPMs fewer than five to seven percent despite their high effectiveness $[3,10,11]$. 
Table 5 Percentage of last pregnancy intended, methods preferred \& reasons for not using LAPMs by married women of Goba town, South East Ethiopia, September 2009

\begin{tabular}{|c|c|c|c|}
\hline Variable & Response & Number & Percent \\
\hline \multirow[t]{2}{*}{ Last pregnancy was planned $\mathrm{n}=677$} & Yes & 543 & 80.2 \\
\hline & No & 134 & 19.8 \\
\hline \multirow[t]{5}{*}{ Method that have been used $n=134$} & Short term & 92 & 68.7 \\
\hline & Natural methods & 9 & 6.7 \\
\hline & IUD & 5 & 3.7 \\
\hline & Norplant & 25 & 18.7 \\
\hline & Tubal ligation & 3 & 2.2 \\
\hline \multirow[t]{2}{*}{ Currently used Short Acting Method preferred $n=410$} & Yes & 375 & 91.4 \\
\hline & No & 35 & 8.6 \\
\hline \multirow[t]{3}{*}{ What LAPM was wanted $\mathrm{n}=35$} & IUD & 7 & 20.0 \\
\hline & Norplant & 24 & 68.6 \\
\hline & Tubal ligation & 4 & 11.4 \\
\hline \multirow[t]{3}{*}{ Reason for not using IUD $n=7$} & Previously used methods inconvenient & 2 & 28.6 \\
\hline & Proven health problem & 4 & 57.1 \\
\hline & Partner/husband opposed & 1 & 14.3 \\
\hline \multirow[t]{3}{*}{ Reason for not tubal ligation $n=4$} & Fear of complications & 3 & 75.0 \\
\hline & Partner/husband opposed & 1 & 25.0 \\
\hline & proven health problem & 6 & 25.0 \\
\hline \multirow[t]{2}{*}{ Reason for not using Norplant $\mathbf{n}=\mathbf{2 4}$} & Fear of complications & 12 & 50.0 \\
\hline & Partner/Husband opposed & 6 & 25.0 \\
\hline
\end{tabular}

The utilization rate of each method in this study was IUD 11 (1.5\%), Norplant 48 (6.5\%) and TL 5 (0.5\%). EDHS 2011 reported the utilization of LAPMs in urban areas was IUD $0.9 \%$, Norplant $3.8 \%$ and female sterilization $1.5 \%$ [2]. Facility based study in Guatemala showed that the utilization rate of the three methods was $6 \%, 8 \%$ and $16 \%$ respectively [9]. This low utilization rate of IUD and Norplant in Goba town could be due to difference in study setting. A study on FP clients in Tehuledre showed that the utilization of Norplant and IUD was 39\% and $8.7 \%$ respectively. The main reason for using LAPMs of contraceptive in Goba was higher for spacing 54\% than limiting $40.6 \%$. This difference could be due to the higher utilization of Voluntary Surgical Contraception (VSC) (52.3\%) in Tehuledre [5]

Although IUDs were the most widely used LAPMs around the world, in this study it was found that the use rate of IUD was very small. IUD can provide effective protection for up to 10 years and could be used as alternative to sterilization for women who may regret after TL [12]. The reasons for non-use of IUD in the town were proven health problem; previously used methods inconvenient and husband / partner opposed. These were also the reasons mentioned in a study in Guatemala [9].

The currently used long acting contraceptive by respondents is higher for Norplant. This is in line with finding of EDHS [2]. Increasing the varieties of implant by incorporating Implanone and Jedelle may increase the opportunity to use LAPMs of contraceptives. They are very safe and convenient; save client time, effort, money, and ease clients load of health facilities as a single visit could provide long duration of protection $[3,13]$. The methods are cost effective and immediately reversible action in case on Norplant and IUD. This makes them the best-suited contraceptive for spacing as well as limiting [3,14-17].

In this study behavioral factors like the number of times discussions on the methods with husband/partner, ever use of any the LAPMs and main decider on using LAPMs were found to be predictors in using LAPMs of contraceptives. This could be due to the reason that those who have frequent discussion with husband/partner, ever used and making decision along with their partner have promotive behavior of using the methods. This could mean there is a need to work in this respect by responsible bodies including service providers.

The highest proportion of unmet need and LAPMs of contraceptive was in the age group 20-39 years. This may indicate that there is still a room for increasing the utilization of LAPMs of contraceptives in the town. In addition, more than half $60.2 \%$ of the respondents were housewife by occupation who may not have access to information and services of reproductive health. 
Table 6 Association of use of LAPMCs and socio-demographic characteristics and others related factors of married women of Goba town, South East Ethiopia, September 2009

\begin{tabular}{|c|c|c|c|c|}
\hline \multirow[t]{2}{*}{ Variable } & \multicolumn{2}{|c|}{ Using LAPMs } & \multirow{2}{*}{$\begin{array}{l}\text { Crude } \\
\text { OR }\end{array}$} & \multirow{2}{*}{$\begin{array}{l}\text { Adjusted } \\
\text { OR }\end{array}$} \\
\hline & Yes (\%) & No (\%) & & \\
\hline \multicolumn{5}{|l|}{ Age } \\
\hline$<30$ years & $26(6.9)$ & $352(93.1)$ & $0.62(0.37,1.04)$ & $0.48(0.16,1.47)$ \\
\hline$\geq 30$ years & $38(10.7)$ & $318(89.3)$ & 1.00 & 1.00 \\
\hline \multicolumn{5}{|l|}{ Number of pregnancy } \\
\hline Zero & $11(5.0)$ & 208(95.0) & $0.52(0.24,1.13)$ & $1.06(0.39,2.90)$ \\
\hline One/Two & $16(8.9)$ & 164(91.1) & $0.96(0.47,1.94)$ & $0.86(0.31,2.38)$ \\
\hline Three/Four & 19(13.6) & $121(86.4)$ & $1.54(0.78,3.06)$ & $0.50(0.18,1.38)$ \\
\hline Five or more & $18(9.2)$ & 177(90.8) & 1.00 & 1.00 \\
\hline \multicolumn{5}{|c|}{ Number of children alive } \\
\hline Zero & $13(5.4)$ & $226(94.6)$ & $0.58(0.27,1.27)$ & $1.50(0.14,16.45)$ \\
\hline One/Two & $21(10.7)$ & $176(89.3)$ & $1.20(0.59,2.45)$ & $0.74(0.15,3.79)$ \\
\hline Three/Four & $16(11.2)$ & $127(88.8)$ & $1.27(0.60,2.70)$ & $0.68(0.17,2.75)$ \\
\hline Five or more & $14(9.0)$ & $141(91.0)$ & 1.00 & 1.00 \\
\hline \multicolumn{5}{|l|}{ Educational status } \\
\hline Illiterate & $4(5.3)$ & 72(94.7) & $0.49(0.17,1.40)$ & $0.93(0.26,3.36)$ \\
\hline Elementary & $8(5.4)$ & 140(94.6) & $0.50(0.23,1,09)$ & $0.77(0.30,2.01)$ \\
\hline High school and above & $52(10.2)$ & $458(89.8)$ & 1.00 & 1.00 \\
\hline \multicolumn{5}{|l|}{ Religion } \\
\hline Muslim & $12(10.2)$ & 149(92.5) & $0.81(0.42,1.55)$ & $* * *$ \\
\hline Christian & $52(9.1)$ & $521(90.9)$ & 1.00 & \\
\hline \multicolumn{5}{|l|}{ Occupation } \\
\hline Government employed & $17(12.6)$ & 118(87.4) & $1.69(0.94,3.05)$ & $1.12(0.51,2.47)$ \\
\hline All others & $47(7.8)$ & $552(92.2$ & 1.00 & 1.00 \\
\hline \multicolumn{5}{|l|}{ Monthly income } \\
\hline$<500$ Ethi. Birr & $31(6.6)$ & 439(93.4) & 1.00 & 1.00 \\
\hline$\geq 500$ Ethi.Birr & $33(12.5)$ & $231(87.5)$ & $2.02(1.21,3.39)$ & $0.64(0.35,1.16)$ \\
\hline \multicolumn{5}{|l|}{ Main decider } \\
\hline Self/Husband & $11(4.2)$ & 249(95.8) & 1.00 & 1.00 \\
\hline Both together & $53(11.2)$ & $421(88.8)$ & $2.85(1.46,5.56)$ & $2.19(1.03,4.65)$ \\
\hline \multicolumn{5}{|l|}{ Number of discussion } \\
\hline Once/Twice & $5(1.6)$ & $310(98.4)$ & 1.00 & 1.00 \\
\hline More often & $59(14.1)$ & $360(85.9)$ & $10.16(4.03,25.64)$ & $4.57(1.72,12.17)$ \\
\hline \multicolumn{5}{|l|}{ Husband opinion } \\
\hline Yes & $59(11.0)$ & $475(89.0)$ & $4.84(1.92,12.25)$ & $0.50(0.15,1.73)$ \\
\hline$\overline{\mathrm{No}}$ & $5(2.5)$ & 195(97.5) & 1.00 & 1.00 \\
\hline \multicolumn{5}{|l|}{ Ever use } \\
\hline Yes & $49(36.6)$ & $85(63.4)$ & $22.48(12.08,41.87)$ & $17.43(9.19,33.03)$ \\
\hline No & $15(2.5)$ & 585(97.5) & 1.00 & 1.00 \\
\hline
\end{tabular}

The unmet need for LAPMs of contraceptives in this study was 69 (9.4\%) which is very much less than the overall modern contraceptive unmet need in Oromia region. EDHS reported unmet need for all methods was $15 \%$. The regional unmet need for contraception was $29.9 \%$. In addition, in this study the unmet need for limiting is higher than the unmet need for spacing. EDHS reported unmet need for spacing was higher than for limiting [2]. USAID reported unmet need for limiting is higher than the unmet need for spacing in many African countries like Ghana, Malawi, Zambia, and Tanzania [18]. The reasons for unmet need for LAPMs were proven health concern, 
Table 7 Association of unmet need for LAPMCs and socio-demographic characteristics and others related factors in married women of Goba town, South East Ethiopia, September 2009

\begin{tabular}{|c|c|c|c|c|}
\hline \multirow[t]{2}{*}{ Variable } & \multicolumn{2}{|c|}{ Unmet need for LAPMs } & \multirow{2}{*}{$\begin{array}{l}\text { Crude } \\
\text { OR }\end{array}$} & \multirow{2}{*}{$\frac{\text { Adjusted }}{\text { Yes (\%) }}$} \\
\hline & Yes (\%) & No (\%) & & \\
\hline$<30$ years & $29(7.7)$ & $349(92.3)$ & $0.66(0.40,1.08)$ & $0.99(0.41,2.39)$ \\
\hline$\geq 30$ years & $40(11.2)$ & $316(88.8)$ & 1.00 & 1.00 \\
\hline \multicolumn{5}{|l|}{ Number of pregnancy } \\
\hline Zero & $11(5.0)$ & 208(95.0) & 1.00 & 1.00 \\
\hline One/Two & $17(9.4)$ & 163(90.6) & $1.97(0.90,4.33)$ & $2.41(0.96,4.78)$ \\
\hline Three/Four & $12(8.6)$ & 128(91.4) & $1.77(0.76,4.14)$ & $1.71(0.72,4.05)$ \\
\hline Five or more & $29(14.9)$ & 166(85.1) & $3.30(1.60,6.81)$ & $3.67(1.75,7.71)$ \\
\hline \multicolumn{5}{|l|}{ Number of children } \\
\hline Zero & $12(5.0)$ & $277(95.0)$ & $0.30(0.15,0.67)$ & $0.25(0.02,2.68)$ \\
\hline One/Two & 18(9.1) & 179(90.9) & $0.61(0.31,1.18)$ & $0.50(0.12,2.29)$ \\
\hline Three/Four & 17(11.9) & $126(88.1)$ & $0.82(0.41,1.61)$ & $1.01(0.34,2.74)$ \\
\hline Five or more & $22(14.2)$ & 133(85.8) & 1.00 & 1.00 \\
\hline \multicolumn{5}{|l|}{ Educational status } \\
\hline Illiterate & $7(9.2)$ & 69(90.8) & $0.93(0.41,2.14)$ & $* * *$ \\
\hline Elementary & $12(8.1)$ & 136(91.9) & $0.81(0.42,1.57)$ & \\
\hline High school and above & $50(9.8)$ & $460(90.2)$ & 1.00 & \\
\hline \multicolumn{5}{|l|}{ Religion } \\
\hline Muslim & $12(7.5)$ & 149(92.5) & $0.73(0.381,1.395)$ & $* * *$ \\
\hline Christian & $57(9.9)$ & $516(90.1)$ & 1.00 & \\
\hline \multicolumn{5}{|l|}{ Occupation } \\
\hline \multicolumn{5}{|l|}{ Government } \\
\hline \multirow[t]{2}{*}{ employed } & & & $1.80(1.02,3.16)$ & $1.64(0.91,2.97)$ \\
\hline & $19(14.1)$ & $116(85.9)$ & & \\
\hline All others & $50(8.3)$ & $549(91.7)$ & 1.00 & 1.00 \\
\hline \multicolumn{5}{|l|}{ Monthly income } \\
\hline$<500$ Ethi. Birr & $44(9.4)$ & 426(90.6) & $0.99(0.59,1.65)$ & $* * *$ \\
\hline$\geq 500$ Ethi.Birr & $25(9.5$ & 239(90.5) & 1.00 & \\
\hline \multicolumn{5}{|l|}{ Number of discussion } \\
\hline Once/Twice & $30(9.5)$ & 285(90.5) & $1.03(0.62,1.69)$ & $* * *$ \\
\hline More often & $39(9.3)$ & $380(90.7)$ & 1.00 & \\
\hline \multicolumn{5}{|l|}{ Intension to use } \\
\hline Yes & $50(16.3)$ & 257(83.7) & $4.13(2.38,7.16)$ & $3.99(2.27,7.02)$ \\
\hline No & $19(4.5)$ & 403(95.5) & 1.00 & 1.00 \\
\hline
\end{tabular}

partner/husband opposition, fear of complication and previous methods were not convenient. In Ethiopia, these and other reasons are the factors for not using LAPMs of contraceptives $[2,4]$. This shows that there is still a need to provide training for providers on LAPMs, involving husbands/ partners in community education on LAPMs of contraceptives, preparing guidelines for providers and client screening for use like in case of IUD insertions. The significant association between grand multigravidas (Five or more pregnancy) and unmet need could mean that there was a need to provide appropriate service to the need of these groups.
LAPMs of contraceptives are also suitable methods for HIV infected individual to reduce mother to child transmission of HIV as they can avoid unintended pregnancy for couples who are living with HIV without restriction $[3,8]$. A study in Addis Ababa showed that people living with HIV also has high-unmet need for contraception [19]. LAPMs could provide safe and effective contraception. Implants are even more effective than IUD in protecting pregnancy. Furthermore, women who had not been pregnant before and breast feeding women from six weeks on wards can use them. Their other advantage is immediate return of fertility after removal [3]. 
Misconception about the use and effectiveness of LAPMs were over came by, transferring the correct knowledge of source and information about their use is of great importance [8]. In the past few months $34.2 \%$ of the respondents have had discussed about LAPMCs and for $71.3 \%$ of them the discussion was made with husband/partner. This may create better opportunity for decision. Use of LAPMs of contraceptives by couples through negotiation and influence of men favors joint decision making on use.

Timing and spacing pregnancies is necessary to improve the outcomes of pregnancy and childbirth for mothers and children. The reasons for not using LAPMs of contraception by those who want to use them include intercourse related, opposition to use, method related and health concern. EDHS and other studies found similar reasons for non-use of LAPMs $[2,4,20]$. This problem could be alleviated through evidence-based policies, guild lines, proper education of husbands, wives, service providers, and promotion of LAPMs of contraceptives through media and community events and community based activities like health extension workers.

\section{Conclusion}

The utilization of LAPMs of contraceptives in the town was higher as compared to the results of other studies and EDHS 2005. Even though there is a good knowledge of LAPMCs in the town, since there are still client with unmet need for LAPMs for both spacing and limiting there is a need to promote the use of LAPMs of contraceptives. The unmet need for LAPMs in the town was indicative of the need to work on unmet need reduction. The reasons mentioned for non use and unmet need for LAPMs of contraception can be managed through proper training of service providers, better counseling skill on family planning, improving method mix, proper management of side effects as they appears and better supply of equipment and commodities.

\section{Competing interests}

The authors' declare that they have no competing interests.

\section{Authors' contributions}

AT has taken a lead role in writing the proposal, submission and follow up for ethical review, data collection, data entry, and writing of the preliminary results. GD, and MY participated in the planning of the study by giving constructive comments and idea. GD and MY have involved significantly in the analysis and writing of the manuscript through commenting. All authors read and approved the final manuscript.

\section{Acknowledgments}

My deepest gratitude goes to Mr. Fekadu Mazengya, Mr. Kassahun Alemu and others for giving me their constructive idea and comments. I also would like to acknowledge staffs of University of Gondar computer center, Goba Town administrative council, Robe Teachers College, Goba Health Science College and Madawalabu University Health Science College for their support in conducting this research project.

\section{Author details}

${ }^{1}$ College of Medicine \& Health Sciences, Madawalabu University, Robe, Bale, Ethiopia. ${ }^{2}$ School of Public Health, Gondar College of Medical Sciences, University of Gondar, Gondar, Ethiopia. ${ }^{3}$ School of Public Health, Gondar of University, Gondar, Ethiopia.

Received: 23 August 2012 Accepted: 23 October 2012

Published: 29 October 2012

\section{References}

1. United Nations Population Fund: International Conference on Population Development. 2005. Accessed on 1/05/2009. Available at: URL http://www.unfpa.org/about.

2. Central Statistical Agency [Ethiopia] and ICF International: Ethiopia Demographic and Health Survey 2011. Addis Ababa, Ethiopia and Calverton, Maryland, USA: Central Statistical Agency and ICF International; 2012.

3. Contraceptive security brief hormonal implants. Accessed on 6/05/2009. Available at: URL: http://deliver.jsi.com/dlvr_content/resources/allpubs/ logisticsbriefs/CSBrie_Hormlmpl.pdf.

4. Peterson Herbert B, Curtis Kathryn M: Long acting methods of contraception. Accessed on 13/06/2009. Available at: URL: http://content.nejm.org/cgi/ content/short/353/20/2169.

5. Asnake M, Walie L, Melkamu $Y$ : Improving the range of contraceptive choices in rural Ethiopia. EJHD 2006, 20(20):74-78.

6. Kebede Y: Contraceptive prevalence and factors associated with usage of contraceptive around Gondar Town. EJHD 2000, 14(3):327-334

7. Merle stop's International Long-acting and permanent methods: MS/'s global impact. Accessed on 6/06/2009 Available at: URL: http://www.mariestopes. org/documents/Longacting20methods20factsheet20FINAL20lo-res.pdf.

8. USDIA and Pathfinder International: Knowledge, Attitude and Practice in PF results from September 2004 survey of Amhara, Oromiya, SNNP and Tigray regions. Accessed on 20/06/2009. Available at: URL:

www: pathFind.org/site/Docserver/CPR survey report- final-June-24pdf.

9. Family health international: A forum for putting knowledge in to practice. Family Health Research. Accessed on 6/06/2009. Available at: URL: http:// www.fhi.org/NR/rdonlyres/evwqxemmr22e4riesd4rjqh7mnvs5y7hghajp2cbck nhgnwanlzwhoyuzlposxnwl7lipybzmxcm7i/LAPMbrief1.pdf.

10. Rosalyn C-DW: Family health International Long-acting and permanent methods of contraception benefit many. Nairobi Kenya: Accessed on 25/05/ 2009. Available at: URL: http://www.fhi.org/NR/rdonlyres/ewei7t4ttwn6km4uc hms3mok336eiotwdytqsnaky2vpwmofcn2kvazlqj62fg3kz265mraemxyjdj/ Mera0803.pdf.

11. Family health international: The case for Long -Acting and Permanent methods [online]. Accessed on 13/05/2009. Available at: URL: http://www.fhi. org/NR/rdonlyres/evwqxemmr22e4riesd4rjqh7mnvs5y7hghajp2cbcknhgn wanlzwhoyuzlposxnwl7lipybzmxcm7i/LAPMbrief1.pdf.

12. Carlos B, Berta T: Availability and acceptability of IUD in Guatemala. 2003. Accessed on 10/06/2009. Available at: URL: http://www.maqweb.org/ iudtoolkit/country_cases/guatemalaiudfinalreport.pdf.

13. Thapa S, De Silva V, G Far M: Potential acceptors of Norplant implants in comparison with recently sterilized women in Sri Lanka Available Family health international and family planning association of Sirlanca. Accessed on 9/05/ 2009. Available at: URL: http://www.popline.org/docs/0943/071438.html.

14. Ortayli N: Users' perspectives on implantable contraceptives for women Accessed on 7/06/2009. Available at: URL: http://www.contraceptionjournal. org/article/s0010-7824(01)00281-5/abstract.

15. Save L, Alleviate P, Spur Development: Invest in Long-acting and permanent Methods of contraception. Advocacy No 1. Accessed on 06/23/2009. Available at: URL: http://www.maqweb.org/iudtoolkit/policies_guidelines/ savelives.pdf.

16. Hall E, Arcanges P: Long acting methods of fertility regulation. Accessed on 11/06/2009. Available at: URL: http://worldpubliclibrary.org/Members.3/ World_Health_Collection/publications/1988/9241561203_(chp9-10).pdf.

17. Jacobstein R: Long-Acting and Permanent Contraception: An International Development, Service Delivery Perspective. Madescape CME. Accessed on 9/06/ 2009. Available at: URL: http://www.medscape.com/viewarticle/56096.

18. Mitchel MJ, Thistle P: Acceptability of levonorgestrel subdermal implants versus tubal ligation for long-term contraception in a rural population of Zimbabwe. Accessed on 20/06/2009. Available at: URL: http://www.contraceptionjournal. org/article/S0010-7824(04)00153-2/abstract. 
19. Emaway D: Demand for family planning among women voluntary counseling and testing clients: The need for integretion, Dessie town North East Ethiopia. Ethiopia: AAU; 2009. Unpublished, J. Joseph Speidel.

20. Joseph SJ, Harper Cynthia C, Shields Wayne C: The potential of long-acting reversible contraception to decrease unintended pregnancy. Accessed on 9/06/ 2009. Available at: URL: http://www.popline.org/docs/1802/328332.html.

doi:10.1186/1742-4755-9-26

Cite this article as: Takele et al: Demand for long acting and permanent methods of contraceptives and factors for non-use among married women of Goba Town, Bale Zone, South East Ethiopia. Reproductive Health 2012 9:26.

\section{Submit your next manuscript to BioMed Central and take full advantage of:}

- Convenient online submission

- Thorough peer review

- No space constraints or color figure charges

- Immediate publication on acceptance

- Inclusion in PubMed, CAS, Scopus and Google Scholar

- Research which is freely available for redistribution 